\title{
Eating Behaviors of American Adults during the COVID-19 Pandemic
}

\author{
Jayanthi Kandiah ${ }^{1}$, Diana Saiki ${ }^{2}$, James A. Jones ${ }^{3}$
}

${ }^{1}$ Department of Nutrition and Health Science, Ball State University, Muncie, USA, ${ }^{2}$ Department of Applied Business Studies, Ball State University, Muncie, USA, ${ }^{3}$ Research and Academic Effectiveness, Ball State University, Muncie, USA

Address for correspondence: Diana Saiki, Department of Applied Business Studies, Ball State University, 206 AT Building, Muncie, USA. E-mail: desaiki@bsu.edu

\section{Abstract}

The objective was to assess the impact of the coronavirus disease (COVID)-19 pandemic on eating behaviors of a cross section of the US adult population. Participants $(n=844)$ completed a survey through Amazon Mechanical Turk which evaluated frequency of consumption of five food categories - mixed dishes, salty, sweet, creamy foods, and beverages during perceived stress. Physical, emotional, and psychological self-care and demographics were also collected. Males eating behaviors especially mixed dishes, sweet foods, and creamy foods decreased when stressed. However, in females, there was an increase in the consumption of sweet and creamy foods. When compared to their counterpart, there was a reduction in food choices among minorities and Hispanics. Participants with higher body mass index had increased eating behaviors of mixed dishes, salty, and sweet foods. The physical self-care scale revealed that there was limited consumption of sweet foods but the psychological self-care demonstrated that there was an increase in creamy food intake.

\section{Introduction}

According to the American Institute of Stress, Hans Selye coined stress as the body's non-specific response to demands caused by any stimuli (i.e., pleasant or unpleasant). Stress occurs when internal (e.g., illness) or external (e.g., environmental or social) factors influence physical and mental abilities to perform affecting the body's homeostasis. ${ }^{[1,2]}$ Perceived stress though multifaceted (e.g., physical, psychological, psychosocial, and medical) is affected by many imaginary and contributing factors which are shaped by societal norms and cultural parameters. ${ }^{[3]}$ According to researchers Yau and Potenza, Allen et al., and Antonyan and Godlevskii, ${ }^{[4-6]}$ examples of stress include psychological (e.g., fluctuations of anxiety), biological (e.g., hormonal), and physiological (e.g., health problem) with the magnitude of experiences ranging from mild to severe. ${ }^{[7]}$ Several methods have been utilized to evaluate stress including assessment of self-reported questionnaires, ${ }^{[8]}$ biochemical assays such as urine, ${ }^{[9]}$ behavioral errors through speech, ${ }^{[10]}$ psychophysiological evaluation of neural changes, ${ }^{[11]}$ and neuroendocrine appraisal of cellular transformations. ${ }^{[12]}$

Stress has been associated with the selection of less healthy foods ${ }^{[4,13,14]}$ and poor eating behaviors ${ }^{[15-17]}$ resulting in higher energy intake, ${ }^{[13,18]}$ increased consumption of fat/saturated fat, ${ }^{[19,20]}$ salt, $^{[21]}$ sugar, ${ }^{[22]}$ and poor diet quality. ${ }^{[14,20]}$ Consumption of a well-balanced healthy diet is one of the major contributors in delaying mortality. Poor dietary habits are precursors for chronic diseases (e.g., obesity, heart disease, stroke, hypertension, diabetes, and cancer) consequently leading to soaring health care costs. ${ }^{[23]}$

There appears to be gender differences in the types of food choices eaten during stressful and non-stressful conditions. When challenged with a solvable (no-stress) or non-solvable (stress) anagrams to solve, Zeller et al. found men ate more significantly unhealthy snacks foods (i.e., potato chips and M\&Ms) when under solvable than during non-solvable conditions. ${ }^{[24]}$ On the contrary,

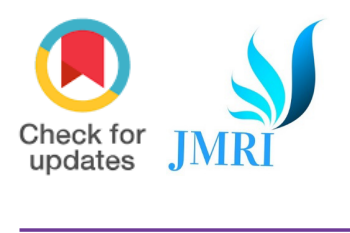

How to cite this article: Kandiah J, Saiki D, Jones J. Eating Behaviors of American Adults during the COVID-19 Pandemic. J Med Res Innov. 2021;5(1):e000248

DOI: $10.32892 /$ jmri.248

Publication history: Received: 24-10-2020 Accepted: 07-01-2021 Published: 08-02-2021

Editor: Dr. Varshil Mehta

Copyright: Kandiah J, Saiki $D$, Jones $J$, This is an open-access article distributed under the terms of the Creative Commons Attribution License CC-BY 4.0., which permits unrestricted use, distribution, and reproduction in any medium, provided the original author and sources are credited.

Funding: NIL

Conflict of Interest: NIL

MK Medkrux 
women had more healthy snack foods (i.e., grapes and peanuts) when faced with solvable anagram to solve but ate more M\&Ms when stressed. ${ }^{[25,26]}$ Similarly, Hbhab found stressed females preferred sweet and high-fat foods and women who were not stressed ate more low-fat foods. ${ }^{[27]}$ When Saiki et al. examined the relationship between food and stress among female university students, there were statistically significant increases in the selection of mixed dishes, salty/crunchy foods, sweet foods, and beverages during perceived stressful conditions. ${ }^{[24]}$ Errisuriz et al. found among a diverse group of students, consumption of unhealthy foods (e.g., soda, coffee, energy drinks, and fast food) was positively associated with perceived stress. ${ }^{[28]}$ Among Australian $1^{\text {st }}$-year university students, though more than $50 \%$ in both genders experienced some degree of stress, it was distinctly more prevalent in women, resulting in them selecting less healthy foods when stressed. ${ }^{[29]}$ Furthermore, earlier research demonstrated that women chose highly palatable or snack foods when under stress using these as a source of comfort. ${ }^{[30]}$ With the coronavirus disease (COVID-19) pandemic in the United Stated in March 2020, lockdowns where enforced nationally for all states. With the exception of essential services (e.g., medical, law enforcement, supermarkets, etc.), all other businesses and institutions including restaurants, and fast food facilities were displaced and employees were compelled to work from home. Since COVID-19 had created a sudden transition from people's normal lifestyle, eating habits, and employment practices, the objective of this research was to assess food choices of Americans during the lived pandemic. Although extensive research has been conducted on stress and eating behaviors, to date, no research has examined a larger population to investigate the influence of perceived stress caused by the COVID-19 pandemic and its influence on food choices of males and females.

The purpose of this study was to assess the impact of the currently lived COVID-19 pandemic on eating behaviors of a cross section of the US adult population.

\section{Method}

A survey was used that included demographic questions (age, sex, race, ethnicity, region of residence, and employment status). To assess eating behaviors, 36 item options were grouped in categories, mixed dishes (e.g., burgers, sandwiches), salty foods (e.g., potato chips, French fries), sweet foods (e.g., ice cream, cookies), creamy foods (e.g., grilled cheese, pasta), and beverages. After the introduction of the research, participants were asked, "For the following questions, identify how frequently did you eat these foods during the current lived COVID-19 pandemic (or within the past month)?" Participants selected from three potential responses for each food item "more during the pandemic - MT," "same as before SAB the pandemic," and "less than before - LB the pandemic," which were coded as 1,0 , and -1 , respectively. In addition, 27 items grouped in three parts of the National Alliance on Mental IIlness' selfcare survey were used to assess physical, emotional, and psychological self-care. To assess stress, an item from $^{[31]} 10$ item perceived stress scale was used: "In the last month, how often have you felt nervous and "stressed"? Participants selected from $0=$ Never to 4 = Very Often with higher score indicating a higher stress level.

Before data collection, the University's Institutional Review Board approved the study as exempt. The survey was made available on Amazon Mechanical Turk during the last week of April 2020. Data were analyzed using SPSS 26. Statistical significance was established at $P<0.05$.

\section{Results}

Of the 1023 surveys completed through Amazon Mechanical Turk, 844 of the participants resided in the United States (US). Mean age for participants was 34.83 (standard deviation [SD] = 11.79) and mean body mass index (BMI) was 25.58 $(S D=5.45)$, with the majority being female $(51.8 \%)$ and working fulltime (56.8\%). Many were working from home due to the pandemic (62.9\%) and few were healthcare workers $(14.2 \%)$. The plurality of respondents was married $(45.6 \%)$, and most were living with family members (71.6\%). The majority of participants were Caucasian (63.2\%), with Asians (22.9\%), African Americans (6.6\%), and multiracial and other (7.3\%). The majority were also nonHispanic (78.0\%).

The internal consistency of the scales was measured by Cronbach's Alpha, with the five eating behavior scales ranging from a low of 0.54 for beverages to a high of 0.80 for sweet foods, with mixed dishes $(0.73)$, creamy foods $(0.73)$, and salty foods scales (0.70) falling in between. The physical self-care scale had an internal consistency coefficient of 0.75 , emotional self-care scale was 0.84 , and 
psychological self-care scale was also 0.84. Three items concerned with fasting, restricted eating, and skipping meals formed a restrictive eating behavior scale with an internal consistency coefficient of 0.71 .

Using a mixed model ANOVA, the five eating behaviors were compared controlling for gender and stress. Interaction effects were found for eating behaviors by gender (Pillai's Trace $=0.01, F$ [4837] $=2.33, P=0.055)$ and eating behaviors by stress
(Pillai's Trace $=0.01, F[4837]=2.43, P=0.047)$, as well as main effects for eating behaviors (Pillai's Trace $=0.17, F[4837]=42.76, P<0.001)$ and gender $(F[1840]=10.33, P=0.001)$. As shown in Figure 1, with zero indicating no change in the eating behavior, mixed dishes showed the most decline overall, particularly for males. Salty food choices also showed some decline, particularly for males. For sweet foods, females or those who were feeling stressed showed increases while those who

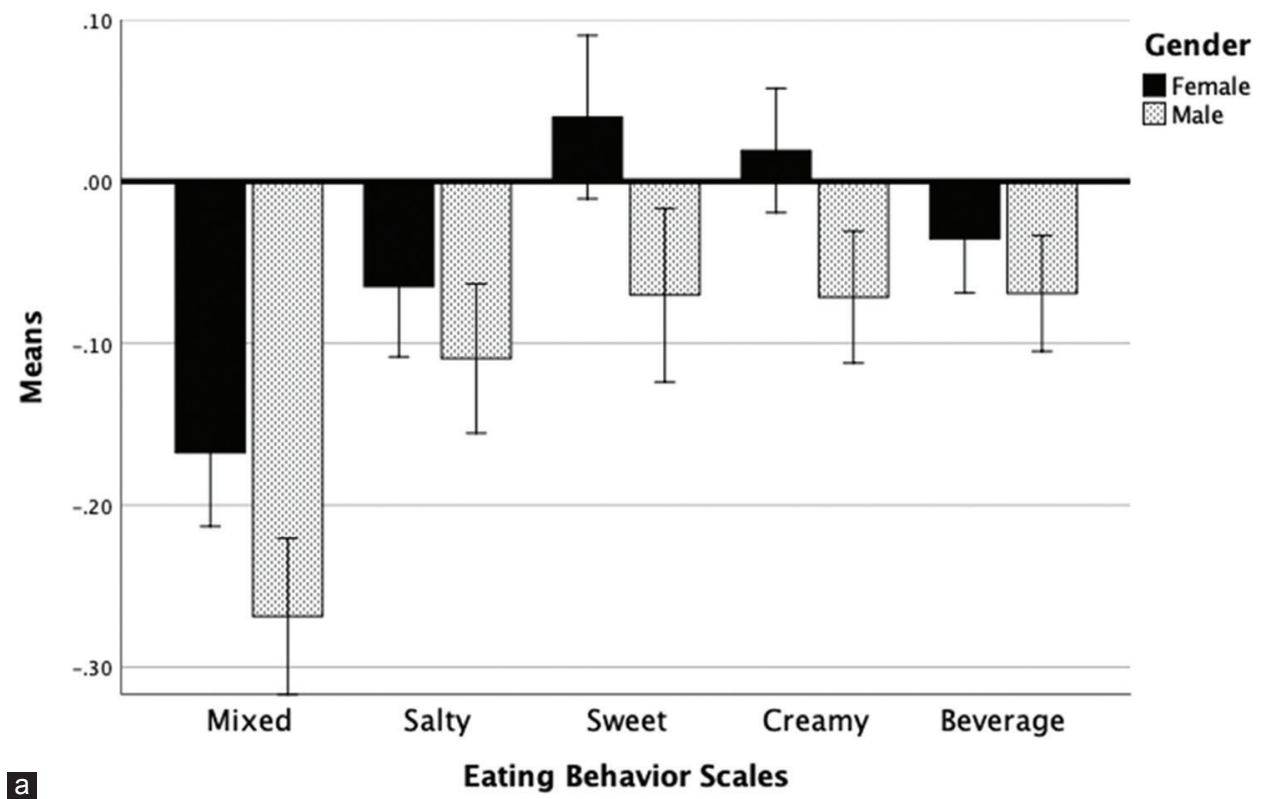

a

Eating Behavior Scales

Error bars: + /- $2 \mathrm{SE}$

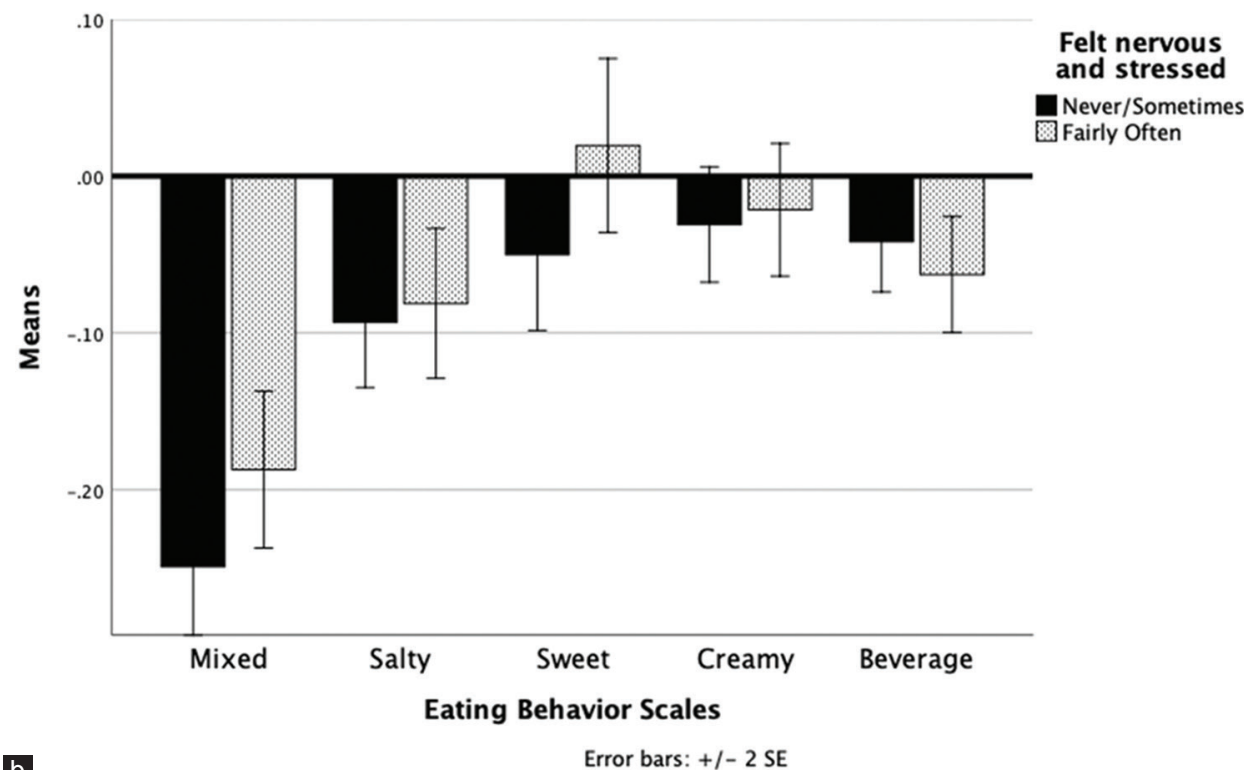

b

Error bars: + /- $2 \mathrm{SE}$

Figure 1: ( $a$ and $b$ ) Eating behavior changes by stress and gender 
were not stressed or male showed declines. Creamy foods had lower means overall except for females, who had an increase. For beverages, all had a small decline with males decreasing the most.

For each eating behavior scale, stress, demographic characteristics, the three self-care scales, and the restrictive eating behavior scale were used as predictors in an ordinary least squares regression analysis. The unstandardized regression coefficients with their standard errors in parentheses are shown in Table 1. All regression models for the eating behavior scales except beverages were found to account for a statistically significant proportion of variance $(P<0.05)$. Excluding beverages, minorities compared to Caucasians reduced their food choices. Hispanics, compared to non-Hispanics, also reduced their choices for mixed dishes, salty foods, and creamy foods. Those with higher BMI values, however, increased on mixed dishes, salty foods, and sweet foods. Males were also found to have decreased their food choices on mixed dishes, sweet foods, and creamy foods. Increased scores on the physical self-care scale were found to be predictive of lower choices for sweet foods, while increased psychological self-care predicted higher levels for creamy foods.

\section{Discussion}

Results from the present study revealed in general when compared to females, males eating behaviors in all food categories decreased when stressed. Unlike males, females exhibited increase eating behaviors of sweet and creamy foods. These observations are in congruent with previous researchers. ${ }^{[24,27]}$ Eating behaviors of females could be explained by the influence of stress on appetite hormones leptin (suppresses huger) and ghrelin (stimulates appetite). Studies on rats and humans have demonstrated that when stressed females exhibited increase ghrelin production and lower levels of the appetite suppressing hormone leptin resulting in diets with higher consumption of sugars, carbohydrates, fat, and calories. In additional, there is supporting evidence unlike males, women have lower levels of neurotransmitter - serotonin, a regulator of mood and appetite. Due to limited serotonin binding sited in the brain, consumption of sugary foods triggers serotonin activity stimulating the releases of endorphins that produce a calming effect during stressful times. ${ }^{[32]}$

Hispanics and minorities reported eating less food. Restrictive eating behaviors could be attributed to

Table 1: Regression coefficients for each eating behavior scale

\begin{tabular}{|c|c|c|c|c|c|}
\hline Predictors & Mixed Dishes & Salty Foods & Sweet Foods & Creamy Foods & Beverages \\
\hline Intercept & $-0.17(0.14)$ & $0.02(0.14)$ & $0.20(0.16)$ & $0.09(0.12)$ & $0.07(0.10)$ \\
\hline Felt nervous and stressed & $0.04(0.04)$ & $0.01(0.04)$ & $0.05(0.04)$ & $0.00(0.03)$ & $0.00(0.03)$ \\
\hline Age & $0.00(0.00)$ & $0.00(0.00)$ & $0.00(0.00)$ & $0.00(0.00)$ & $0.00(0.00)$ \\
\hline Employment status & $0.04(0.03)$ & $0.02(0.02)$ & $0.01(0.03)$ & $-0.01(0.02)$ & $0.01(0.02)$ \\
\hline Caucasian vs. Minority & $-0.23 *(0.04)$ & $-0.11 *(0.04)$ & $-0.13 *(0.04)$ & $-0.12 *(0.03)$ & $-0.05(0.03)$ \\
\hline Hispanic & $-0.11 *(.04)$ & $-0.13 *(0.04)$ & $-0.08(0.05)$ & $-0.11(0.04)$ & $-0.05(0.03)$ \\
\hline $\mathrm{BMI}$ & $0.01 *(0.00)$ & $0.00(0.00)$ & $0.01 *(0.00)$ & $0.00(0.00)$ & $0.00(0.00)$ \\
\hline Marital status & $0.02(0.04)$ & $-0.03(0.04)$ & $0.01(0.05)$ & $0.02(0.04)$ & $-0.04(0.03)$ \\
\hline Live with others & $0.05(0.05)$ & $0.01(0.05)$ & $0.00(0.06)$ & $0.00(0.04)$ & $0.02(0.04)$ \\
\hline Healthcare worker & $0.03(0.05)$ & $0.04(0.05)$ & $-0.01(0.06)$ & $-0.04(0.05)$ & $0.01(0.04)$ \\
\hline Working from home & $-0.01(0.04)$ & $0.07(0.04)$ & $0.03(0.05)$ & $0.05(0.04)$ & $0.05(0.03)$ \\
\hline Gender & $-0.10 *(0.04)$ & $-0.06(0.04)$ & $-0.14 *(0.04)$ & $-0.09 *(0.03)$ & $-0.05(0.03)$ \\
\hline Physical SC & $-0.05(0.04)$ & $-0.05(0.04)$ & $-0.12 *(0.05)$ & $-0.07(0.04)$ & $-0.02(0.03)$ \\
\hline Psychological SC & $-0.02(0.04)$ & $0.07(0.04)$ & $-0.03(0.05)$ & $0.07 *(0.03)$ & $0.01(0.03)$ \\
\hline Emotional SC & $-0.03(0.04)$ & $-0.06(0.04)$ & $-0.01(0.04)$ & $-0.05(0.03)$ & $0.01(0.03)$ \\
\hline Restrictive eating & $0.01(0.03)$ & $-0.05(0.03)$ & $-0.07(0.04)$ & $0.02(0.03)$ & $0.02(0.02)$ \\
\hline $\mathrm{R}^{2}$ & $0.12 *$ & $0.06 *$ & $0.08 *$ & $0.07 *$ & 0.03 \\
\hline
\end{tabular}

${ }^{*} P<0.05$. Standard error of regression coefficient shown in parentheses 


\section{Kandiah, et al.: Eating behaviors during covid-19}

participants' social determinants to health, level of affluence to purchase non-seasonal foods with higher nutrient content, loss of employment or job opportunities, and social distancing during the lockdown of the pandemic. ${ }^{[33]}$ Furthermore, eating of less foods could be due to participants' fear of getting ill and/or the unavailability or limited insurance coverage for health care services. It has been consistently observed across all ages, when compared to non-Hispanic whites, African Americans, and people of Hispanic origin persistently have lower insurance coverage. ${ }^{[34]}$

This research showed that people with higher BMI had increased eating behaviors of mixed dishes, salty, and sweet foods. Overweight and obese individuals have been reported to be most vulnerable to stress-related eating behaviors, often times craving hyperpalatable foods (e.g., snacks, desserts) in the absence of hunger as a mechanism to regulate and suppress stress. ${ }^{[35,36]}$ A possible speculation for the reduction in beverages across both genders could be their confinement of being in their residence, lack of physical activity, and not being outdoors or limited availability of an assortment of beverages at home. Increased scores on the physical self-care scale were found to be predictive of lower choices for sweet foods, while increased psychological selfcare predicted higher levels for creamy foods. Sweet foods choices on this survey are considered unhealthy, therefore, during the pandemic participants who were aware of their physical selfcare (eating healthy foods, exercising, take time away from telephones, wear clothing you like, etc.) were aware of limiting sweet foods. Perhaps staying at home allowed these participants to have more control over their food intake and availability by grocery shopping as opposed to eating out. Intake of creamy foods increased with psychological selfcare. Creamy foods are associated with comfort. There is evidence that these foods can help sooth patients in distress. ${ }^{[37]}$ Perhaps eating creamy foods was part of increasing psychological care including such variables as noticing inner thoughts, respecting one's limitations, and coping with stress.

\section{Conclusion}

Some of the strengths of this research included assessment of eating behaviors of a cross-section of the US adult population during a unique time frame, that is, occurrence of the COVID-19 pandemic, data were gathered during a rare perceived stressful time, eating behaviors were grouped into five food categories, and a reliable instrument was used to assess physical, emotional, and psychological self-care. Like any study, some minor limitations included data collection using Amazon Turk. Although the pandemic occurred globally, data collection occurred just in the United States. Recommendations for future research include comparing this data with post-COVID period, investigation of a global population, examining alterations in eating behaviors across demographics (e.g., age, ethnicity, income, employment, etc.), and possibly implication of poor eating behaviors on overall health status. In conclusion, our research suggests stress in males demonstrated decreased eating behaviors in all food categories but influenced increased intake of sweet and creamy foods in females.

\section{References}

1. Choi J. Healthy bone tissue homeostasis. Exp Mol Med 2020;52:1165.

2. O'Connor DB, Ferguson E. Stress and stressors. In: Benyamini $\mathrm{Y}$, Johnston $\mathrm{M}$, Karademas EC, editors. Assessment in Health Psychology. Göttingen, Boston: Hogrefe; 2016. p. 104-18.

3. Moore KA, Cooper CL. Stress in mental health professionals: A theoretical overview. Int J Soc Psychiatry 1996;42:82-9.

4. Yau YH, Potenza MN. Stress and eating behaviors. Minerva Endocrinol 2013;38:255.

5. Allen AP, Kennedy PJ, Cryan JF, Dinan TG, Clarke G. Biological and psychological markers of stress in humans: Focus on the trier social stress test. Neurosci Biobehav Rev 2014;38:94-124.

6. Antonyan IS, Godlevskii LS. Role of dopaminergic mechanisms in the formation of stress-induced ulcer damages to the duodenum and stomach in rats. Neurophysiology 2012;44:79-82.

7. Stults-Kolehmainen MA, Sinha R. The effects of stress on physical activity and exercise. Sports Med 2014;44:81-121.

8. Garrett N. 2013 Jin Shin Jyutsu ${ }^{\circledR}$ Patient Response, DNP Projects No. 56; 2015. Available from: https:// www.uknowledge.uky.edu/dnp_etds/56. [Last accessed on 2021 Feb 22].

9. Ferguson KK, McElrath TF, Chen YH, Mukherjee B, Meeker JD. Urinary phthalate metabolites and biomarkers of oxidative stress in pregnant women: A repeated measures analysis. Environ Health Perspect 2015;123:210-6.

10. Laures-Gore JS, DuBay MF, Duff MC, Buchanan TW. Identifying behavioral measures of stress in individuals with aphasia. J Speech Lang Hear Res 2010;53:1394-400.

11. Putman P, Verkuil B, Arias-Garcia E, Pantazi I,

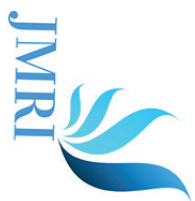


van Schie C. EEG theta/beta ratio as a potential biomarker for attentional control and resilience against deleterious effects of stress on attention. Cogn Affect Behav Neurosci 2014;14:782-91.

12. Baum A, Grunberg NE, Singer JE. The use of psychological and neuroendocrinological measurements in the study of stress. Health Psychol 1982;1:217.

13. Laugero KD, Falcon LM, Tucker KL. relationship between perceived stress and dietary and activity patterns in older adults participating in the Boston Puerto Rican health study. Appetite 2011;56:194-204.

14. Richardson AS, Arsenault JE, Cates SC, Muth MK. Perceived stress, unhealthy eating behaviors, and severe obesity in low-income women. Nutr J 2015;14:122.

15. Torres SJ, Nowson CA. Relationship between stress, eating behavior, and obesity. Nutrition 2007;23:887-94.

16. Block JP, He Y, Zaslavsky AM, Ding L, Ayanian JZ. Psychosocial stress and change in weight among US adults. Am J Epidemiol 2009;170:181-92.

17. Pasquali R. The hypothalamic-pituitary-adrenal axis and sex hormones in chronic stress and obesity: Pathophysiological and clinical aspects. Ann N Y Acad Sci 2012;64:20-35.

18. Jordan TR, Khubchandani J, Wiblishauser M. The impact of perceived stress and coping adequacy on the health of nurses: A pilot investigation. Nurs Res Pract 2016;2016:5843256.

19. Vidal EJ, Alvarez D, Martinez-Velarde D, VidalDamas L, Yuncar-Rojas KA, Julca-Malca A, et al. Perceived stress and high fat intake: A study in a sample of undergraduate students. PLoS One 2018;13:e0192827.

20. Ulrich-Lai YM, Fulton S, Wilson M, Petrovich G, Rinaman L. Stress exposure, food intake and emotional state. Stress 2015;18:381-99.

21. Torres SJ, Turner Al, Nowson CA. Does stress induce salt intake? Br J Nutr 2010;103:1562-8.

22. Knüppel A, Shipley MJ, Llewellyn $\mathrm{CH}$, Brunner EJ. Sugar intake from sweet food and beverages, common mental disorder and depression: Prospective findings from the Whitehall II study. Sci Rep 2017;7:6287.

23. Micha R, Peñalvo JL, Cudhea F, Imamura F, Rehm CD, Mozaffarian D. Association between dietary factors and mortality from heart disease, stroke, and Type 2 diabetes in the United States. JAMA 2017;317:912-24.

24. Saiki DE, Kandiah J, McCarthy L. Women's perceived influence of stress on their dressing and eating behaviors. Int J Home Econ 2012;5:279-89.

25. Zellner DA, Saito $S$, Gonzalez J. The effect of stress on men's food selection. Appetite 2007;49:696-9.

26. Zellner DA, Loaiza S, Gonzalez Z, Pita J, Morales J, Pecora $D$, et al. Food selection changes under stress. Physiol Behav 2006;87:789-93.

27. Habhab S, Sheldon JP, Loeb RC. The relationship between stress, dietary restraint, and food preferences in women. Appetite 2009;52:437-44.

28. Errisuriz VL, Pasch KE, Perry CL. Perceived stress and dietary choices: The moderating role of stress management. Eat Behav 2016;22:211-6.

29. Papier K, Ahmed F, Lee P, Wiseman J. Stress and dietary behaviour among first-year university students in Australia: Sex differences. Nutrition 2015;31:324-30.

30. Wallis DJ, Hetherington MM. Emotions and eating. Self-reported and experimentally induced changes in food intake under stress. Appetite 2009;52:355-62.

31. Cohen S, Kamarck T, Mermelstein R. A global measure of perceived stress. J Health Soc Behav 1983;24:385-96.

32. Karolinska Institute. Sex Differences in the Brain's Serotonin System, Medical Xpress-Medical Research Advances and Health News, Medical Xpress; 2008 Available from: https://www.medicalxpress.com/ news/2008-02-sex-differences-brain-serotonin. html. [Last accessed on 2020 Oct 21].

33. Bonaccio $M$, di Castelnuovo A, Pounis G, Costanzo S, Persichillo M, Cerletti C, et al. High adherence to the Mediterranean diet is associated with cardiovascular protection in higher but not in lower socioeconomic groups: Prospective findings from the Moli-Sani study. Int J Epidemiol 2017;46:1478-87.

34. Sohn H. Racial and ethnic disparities in health insurance coverage: Dynamics of gaining and losing coverage over the life-course. Popul Res Policy Rev 2017;36:181-201.

35. Lemmens SG, Rutters F, Born JM, WesterterpPlantenga MS. Stress augments food 'wanting' and energy intake in visceral overweight subjects in the absence of hunger. Physiol Behav 2011;103:157-63.

36. Laitinen J, Ek E, Sovio U. Stress-related eating and drinking behavior and body mass index and predictors of this behavior. Prev Med 2002;34:29-39.

37. Wendin K, Ekman S, Bülow M, Ekberg O, Johansson D, Rothenberg $\mathrm{E}$, et al. Objective and quantitative definitions of modified food textures based on sensory and rheological methodology. Food Nutr Res 2010;54:5134. 